\title{
Tratamento endodôntico conservador em lesão periapical extensa asséptica: Relato
}

\author{
de caso \\ Conservative endodontic treatment in extensive periapical lesion: Case report \\ Tratamiento endodôntico conservador em lesión asséptica extensa: Reporte de caso
}

Recebido: 12/04/2021 | Revisado: 19/04/2021 | Aceito: 23/04/2021 | Publicado: 13/05/2021

\author{
Rosana Maria Coelho Travassos \\ ORCID: https://orcid.org/0000-0003-4148-1288 \\ Universidade de Pernambuco, Brasil \\ E-mail: travassos.rosana@gmail.com \\ Jhony Herick Cavalcanti Nunes Negreiros \\ ORCID: https://orcid.org/0000-0002-3407-1021 \\ Universidade Federal de Pernambuco, Brasil \\ E-mail: jhonyherick@gmail.com \\ Joás de Araújo Teixeira \\ ORCID: https://orcid.org/0000-0002-9372-0159 \\ Universidade Federal de Pernambuco, Brasil \\ E-mail: joasaraujo@hotmail.com \\ Maria Carlla Aroucha Lyra \\ ORCID: https://orcid.org/0000-0002-1974-4427 \\ Universidade Federal de Pernambuco, Brasil \\ E-mail: mcarllalyra@gmail.com \\ Lívia Mirelle Barbosa \\ ORCID: https://orcid.org/0000-0002-8992-2890 \\ Universidade Federal de Pernambuco, Brasil \\ E-mail: dra.liviabarbosa@gmail.com \\ Osman Jucá Rêgo Lima Netto \\ ORCID: https://orcid.org/0000-0003-0159-7382 \\ Universidade Federal de Pernambuco, Brasil \\ E-mail: osmanjuca@hotmail.com
}

\begin{abstract}
Resumo
Embora o fator etiológico mais frequente de injúria pulpar seja a presença de microrganismos que estabelecem um quadro infeccioso, uma polpa injuriada (assepticamente) por traumatismo torna-se mais sensível às bactérias infectantes que uma polpa dental saudável. O caso clínico apresentado neste trabalho descreve o sucesso de um tratamento endodôntico conservador de um elemento dentário necrosado por trauma e apresentando extensa lesão periapical crônica. Após a realização do acesso, limpeza do conduto e implementação do curativo de demora à base de pasta de hidróxido de cálcio, ocorreu a reagudização expressiva da lesão. Porém, o callen associado ao PMCC apresentou eficácia para o tratamento da lesão quando adotado como segunda escolha para a medicação intracanal. Após a obturação do canal radicular pela técnica Híbrida de Tagger, foi realizada a proservação do caso durante 1 ano, constatando-se radiograficamente a remissão da lesão. O sucesso do tratamento deste caso esteve diretamente relacionado ao correto diagnóstico que permitiu o estabelecimento da melhor conduta. Abordagens mais conservadoras devem ser a primeira escolha do profissional mesmo diante de casos complexos, como o apresentado.

Palavras-chave: Endodontia; Tratamento conservador; Necrose da polpa dentária.
\end{abstract}

\begin{abstract}
Embora o fator etiológico mais frequente de injúria pulpar seja a presença de microrganismos que estabelecem um quadro infeccioso, uma polpa injuriada (assepticamente) por traumatismo torna-se mais sensível às bactérias infectantes que uma polpa dental saudável. O caso clínico apresentado neste trabalho descreve o sucesso de um tratamento endodôntico conservador de um elemento dentário necrosado por trauma e apresentando extensa lesão periapical crônica. Após a realização do acesso, limpeza do conduto e implementação do curativo de demora à base de pasta de hidróxido de cálcio, ocorreu a reagudização expressiva da lesão. Porém, o Callen associado ao PMCC apresentou eficácia para o tratamento da lesão quando adotado como segunda escolha para a medicação intracanal. Após a obturação do canal radicular pela técnica Híbrida de Tagger, foi realizada a proservação do caso durante 1 ano, constatando-se radiograficamente a remissão da lesão. O sucesso do tratamento deste caso esteve diretamente relacionado ao correto diagnóstico que permitiu o estabelecimento da melhor conduta. Abordagens mais conservadoras devem ser a primeira escolha do profissional mesmo diante de casos complexos, como o apresentado.
\end{abstract}

Keywords: Endodontics; Conservative treatment; Dental pulp necrosis. 


\begin{abstract}
Resumen
Aunque el factor etiológico más frecuente de lesión pulpar es la presencia de microorganismos que establecen una condición infecciosa, una pulpa lesionada (asépticamente) por trauma se vuelve más sensible a las bacterias infecciosas que una pulpa dental sana. El caso clínico presentado en este trabajo describe el éxito de un tratamiento endodóntico conservador de un elemento dentario necrótico por trauma y que presenta una extensa lesión periapical crónica. Tras el acceso, limpieza del conducto e implantación del apósito retardador a base de pasta de hidróxido de calcio, se produjo un reordenamiento importante de la lesión. Sin embargo, el callen asociado a PMCC mostró eficacia para el tratamiento de la lesión cuando se adoptó como la segunda opción para la medicación intracanal. Tras el llenado del conducto radicular mediante la técnica Tagger Hybrid, el caso se conservó durante 1 año, con evidencia radiográfica de remisión de la lesión. El tratamiento exitoso de este caso estuvo directamente relacionado con el diagnóstico correcto que permitió establecer el mejor abordaje. Los enfoques más conservadores deben ser la primera opción del profesional incluso ante casos complejos, como el presentado.
\end{abstract}

Palabras clave: Endodoncia; Tratamiento conservador; Necrosis de la pulpa dental.

\title{
1. Introdução
}

Um dos principais objetivos da endodontia é promover o reparo tecidual através da sanificação do sistema de canais radiculares, através de instrumentos mecânicos (limas) e irrigação com substâncias químicas auxiliares (SQA). Para alcançar o sucesso da terapia endodôntica, todas as etapas devem ser realizadas de forma criteriosa, desde o diagnóstico até a obturação dos canais (Oliveira, et al., 2020; Arashiro, et al., 2013).

O preparo biomecânico dos canais radiculares e a utilização das soluções irrigadoras proporcionam uma significativa redução no número de microorganismos presentes no interior do sistema de canais radiculares. Porém, em alguns casos, se faz necessário o emprego da medicação intracanal (MIC) entre sessões, com o objetivo de potencializar o processo de sanificação do sistema de canais radiculares e com isso favorecer o processo de reparo periapical. A MIC deve apresentar potencial antimicrobiano, ser biocompatível e estimular a reparação tecidual (Vera, et al., 2012).

Os traumas dentoalveolares, em dentes permanentes, são um problema de saúde pública (Petti, et al., 2018). Podem causar desde fraturas de esmalte à danos mais extensos, como a fratura de osso alveolar. Entre as sequelas decorrentes do trauma dental, a necrose pulpar é a mais prevalente e está relacionada ao comprometimento do feixe vásculo-nervoso, por inflamação pulpar, pressão e tensão dos nervos apicais (Yamashita, et al., 2017).

As lesões traumáticas não seguem a progressão tradicional e podem ocorrer diretamente em qualquer nível dos tecidos dentais ou perirradiculares. Assim, podem ocorrer diretamente na polpa, no cemento, no ápice da raiz, no ligamento periodontal ou no osso adjacente, de forma individual ou combinada (Ricucci, et al., 2015; Fouad, 2019).

As intervenções endodônticas após lesões traumáticas são contempladas após um período de observação quando o dente mostra sinais e sintomas de necrose pulpar. Durante esse período de retardo, mais invasão bacteriana pode ocorrer, levando ao surgimento de lesões periapicais e reabsorções (Fouad, 2019).

Lesões periapicais extensas podem ocorrer em dentes traumatizados e definir sua conduta terapêutica é extremamente desafiador. Muitos profissionais consideram a intervenção cirúrgica como primeira escolha de tratamento. No entanto, atualmente, há uma tendência para se optar por tratamentos conservadores, reservando as intervenções cirúrgicas para casos em que a lesão persiste (Mitra, et al., 2017).

Portanto, o objetivo do presente artigo é descrever um caso clínico de uma extensa lesão periapical decorrente de trauma dental, tratada por uma abordagem endodôntica não cirúrgica, com expressivo reparo periapical.

\section{Metodologia}

O presente artigo trata-se de um relato de caso clínico, descritivo e qualitativo, em que se observa o tratamento conservador de uma lesão periapical extensa asséptica. Quanto aos termos éticos, o paciente assinou o Termo de consentimento Livre e Esclarecido e foram respeitados os princípios éticos descritos na Declaração de Helsinque (Pereira et al., 2018). 


\section{Relato de Caso Clínico}

Paciente do sexo masculino, 37 anos, compareceu à Clínica do Curso de Especialização em Endodontia do Centro de Pós-Graduação em Odontologia, Recife - Pernambuco, com queixa de dor e aumento de volume ocasional na região mentoniana.

$\mathrm{Na}$ anamnese, o paciente relatou história de trauma na região do mento há aproximadamente três anos e que o aumento de volume se dava periodicamente, mas não sabia relatar acerca do intervalo entre um episódio e outro. Relatou que buscou por atendimento odontológico prévio, mas que não houve resolutividade do caso. Ademais, o paciente relatou não ter nenhum problema de saúde e que não fazia uso ou tinha alergia a fármacos. Ao exame físico, foi observado que os dentes anteriores inferiores estavam hígidos, com ausência de biofilme ou tártaro visíveis à olho nu, e presença de discreto edema difuso na região de fundo de vestíbulo e região mentoniana. Os testes de percussão (horizontal e lateral) e de sensibilidade, ao frio e ao calor, foram realizados a fim de se verificar a vitalidade pulpar e as condições do tecidos perirradiculares dos incisivos e caninos inferiores. Foram encontrados parâmetros de normalidade com relação aos testes e o diagnóstico de vitalidade pulpar foi constatado para todos os dentes, exceto para o incisivo central inferior esquerdo (dente 31), tido para este o diagnóstico de polpa necrosada.

Radiograficamente, foi constatada radiotransparência óssea extensa difusa e irregular, com contornos indefinidos, que se estendia aos ápices dentais dos dentes 32, 31, 41 e 42 (Figura 1).

Figura 1. Radiografia periapical inicial.

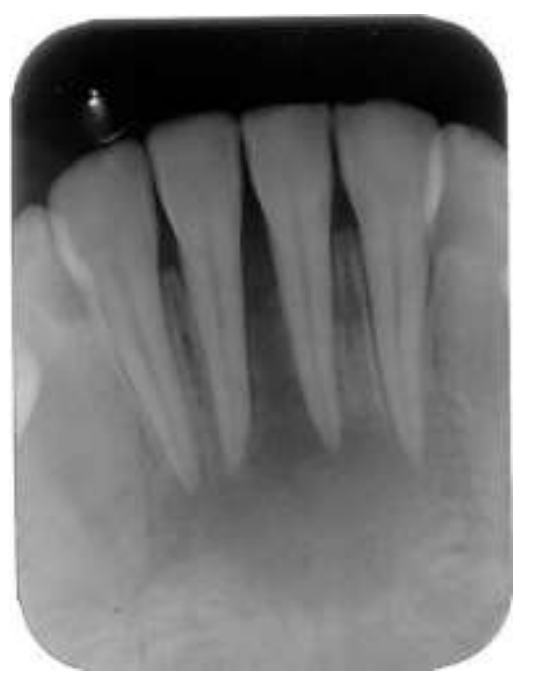

Fonte: Autores.

A partir da avaliação do caso e realização do plano de tratamento, foi instituído o tratamento endodôntico do dente 31 através da técnica coroa-ápice sem pressão. Pela condição da região e do dente, foi optado por não realizar anestesia local e a abertura coronária foi procedida com ponta diamantada 1012. Ao fim deste passo, foi observado restos pulpares necrosados e ausência de sangramento coronário, como esperado. A partir da radiografia periapical inicial, foram determinados os valores do comprimento aparente do dente (CAD), comprimento real do instrumento (CRI), comprimento parcial de trabalho (CPT) e comprimento para a agulha de irrigação do canal. Para o CAD, foi constatado $19 \mathrm{~mm}$ e, assim, 17mm para o CRI (CAD-2mm). Para o CPT, o resultado foi de $15 \mathrm{~mm}$ (CAD-4mm) e, para o comprimento da agulha de irrigação, $14 \mathrm{~mm}(\mathrm{CAD}-5 \mathrm{~mm})$.

Logo após, foi utilizada uma seringa de $5 \mathrm{ml}$ e agulha Navitip 30ga (Ultradent@) para a irrigação com $5 \mathrm{ml}$ com hipoclorito de sódio $(\mathrm{NaCl})$ 2,5\% de formulação pronta para uso. A exploração do canal radicular foi realizada com a lima $\mathrm{K}$ File número \#15 na medida do CRI e utilizadas as brocas Gattes-Glidden de números 4, 3 e 2 no terço cervical e médio na 
medida do CPT, a fim de se remover o tecido pulpar necrosado. A título informativo, uma Gates-Glidden de número 2 tem correspondência a uma lima do tipo K de número \#70.

A odontometria foi realizada e, assim, determinada a distância ápice-instrumento (DAI) de 1mm. Através do DAI, o comprimento real do dente (CRD) pôde ser calculado através da fórmula CRD = CRI+/-DAI, resultando no valor de $20 \mathrm{~mm}$. O comprimento real de trabalho (CRT) foi de $19 \mathrm{~mm}$. A ampliação do canal foi realizada progressivamente iniciando-se com uma lima K-File \#60 até \#30, com irrigação de $5 \mathrm{ml}$ de $\mathrm{NaOCl}$ 2,5\% a cada troca de instrumento.

Após secagem do canal radicular, foi introduzida a pasta de hidróxido de cálcio UltraCal XS (SSWhite@), sendo comprovado o preenchimento com a medicação intracanal radiograficamente (Figura 2). Para selamento coronário, foi utilizado cimento de ionômero de vidro para restauração (Maxxion C, FGM).

Figura 2. Comprovação radiográfica do preenchimento do canal pelo hidróxido de cálcio.

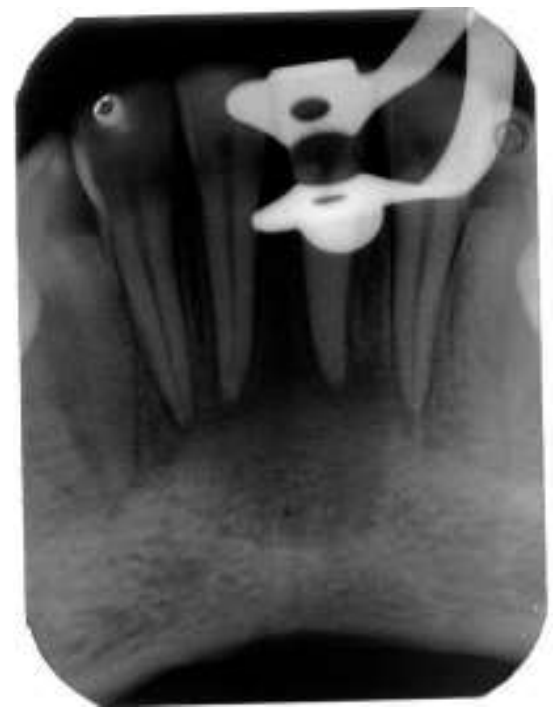

Fonte: Autores.

Após 8 dias, o paciente retornou ao atendimento apresentando reagudização do edema mentoniano de forma mais acentuada (Figuras 3A e B), dor aguda de grau 8 na escala visual analógica (EVA) e drenagem espontânea do exsudato através do sulco gengival (Figura 3C). A drenagem foi realizada intraoralmente realizando-se uma incisão intrasulcular com o auxílio de bisturi (lâmina 15C). Foi prescrito, por via oral, 1 comprimido de amoxicilina $875 \mathrm{mg}$ associado a clavulanato de potássio $125 \mathrm{mg}$ a cada 12 horas por 7 dias e, por via sublingual, 1 comprimido de trometamol cetorolaco 10 mg a cada 8 horas por 3 dias.

Figura 3. A - Aspecto extraoral do edema. B - Aspecto intraoral do edema. C - Drenagem sulcular do exsudato.
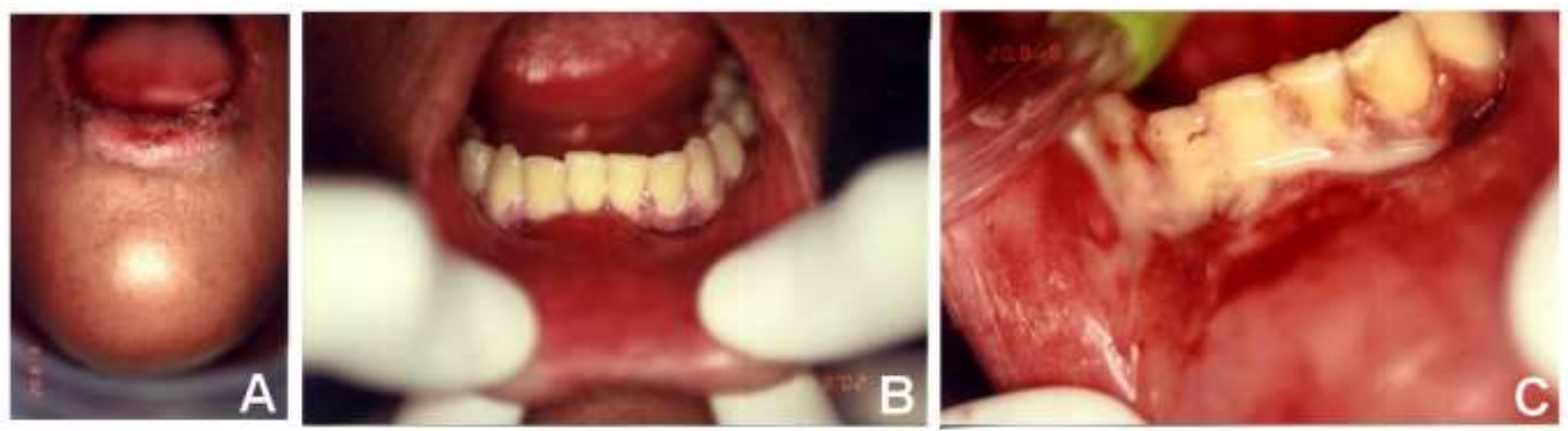

Fonte: Autores. 
Foi pedido para que o paciente retornasse após 8 dias e foi observado o desaparecimento dos sinais e sintomas clínicos relacionados à dor e ao edema. Nova neutralização do conduto séptico/tóxico foi realizada e, como nova medicação intracanal, foi utilizado o Calen associado ao PMCC (SS White ${ }^{\mathrm{TM}}$ ), preenchendo-se todo o canal radicular, o qual permaneceu no conduto radicular por 30 dias. Foi solicitado que o paciente retornasse após este tempo para realização da obturação do canal radicular pela técnica híbrida de Tagger e cimento endodôntico à base de hidróxido de cálcio (Sealapex, KerrTM) (Figuras 4A e B). O selamento da câmara pulpar foi realizado com cimento de ionômero de vidro para restauração (Maxxion C, FGM) e restauração final em resina composta (Filtek Z250 XT, 3M $\mathrm{M}^{\mathrm{TM}}$ ).

Consultas de proservação foram solicitadas e realizadas após 6 meses e 1 ano (Figuras 5A e B). Foi observado radiograficamente reparo ósseo significativo da lesão aos 6 meses, com seu desaparecimento completo após 1 ano. Ademais, o paciente afirmou não ter tido nenhum episódio de dor ou edema durante este período de tempo, o que corrobora e afirma o sucesso do tratamento endodôntico conservador instaurado.

Figuras 4. A - Comprovação da condensação lateral. B - Obturação do canal radicular.
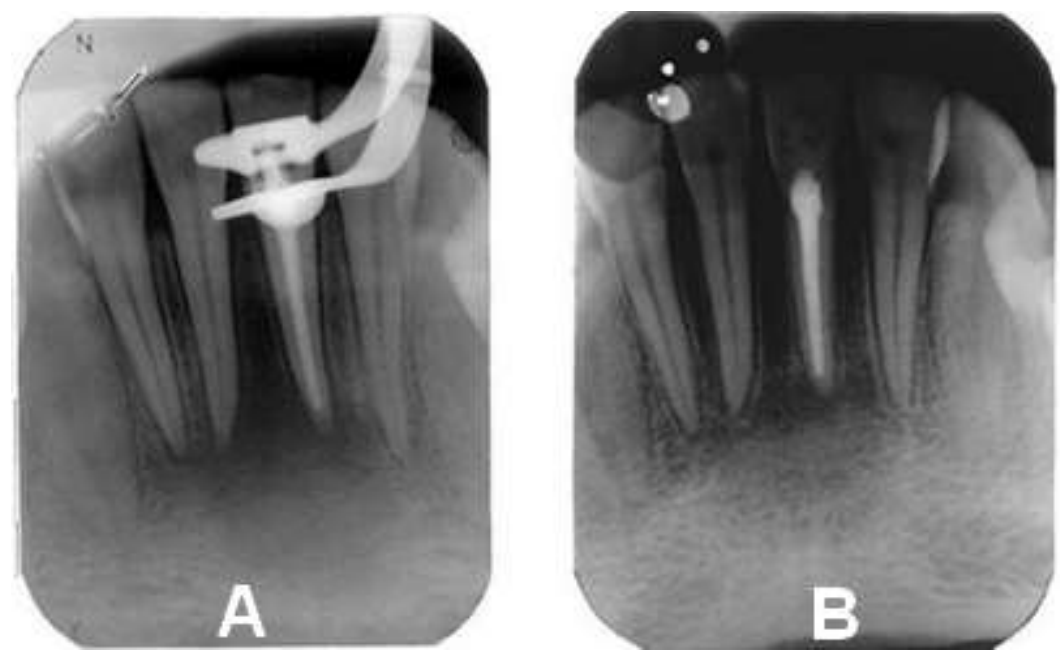

Fonte: Autores.

Figuras 5. A - Proservação após 6 meses. B - Proservação após 1 ano.
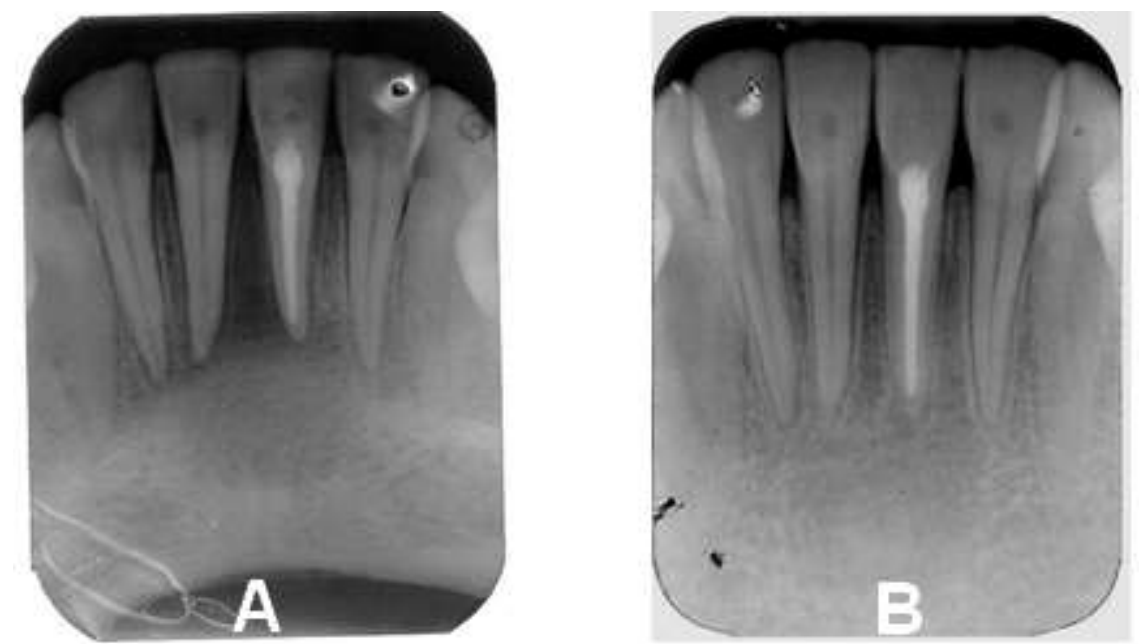

Fonte: Autores. 


\section{Discussão}

A lesão apresentada neste caso foi decorrente de trauma na região do mento ocorrida há três anos do início do tratamento. A energia do impacto aplicada ao mento originou uma lesão de origem asséptica envolvendo a região dos dentes anteriores inferiores cuja causa, até então, era desconhecida. Dessa forma, a condução do exame clínico é muito importante e a anamnese não pode ser subestimada, pois é neste momento que o paciente relata informações preciosas sobre a história clínica da doença, no qual surge o diagnóstico presuntivo, confirmado posteriormente pelos exames complementares.

O trauma dentoalveolar é capaz de causar obliteração do canal pulpar e reabsorção radicular inflamatória, originando o processo de necrose pulpar (Andreasen, et al., 2015) e, por este motivo, o acompanhamento do caso pelo cirurgião-dentista é necessário para estabelecer o tratamento adequado a fim de evitar complicações e agravamento, como no relato deste caso.

Após a realização do acesso, limpeza do conduto e implementação do curativo de demora, ocorreu a reagudização expressiva da lesão devido, provavelmente, ao trauma mecânico da instrumentação ou à contaminação por novos patógenos aeróbios, mesmo sob isolamento absoluto e protocolo de irrigação adequado. No entanto, após a troca do curativo de demora por Calen associado ao PMCC houve regressão do processo infeccioso/inflamatório, permitindo a finalização do tratamento.

Como medicação intracanal, o hidróxido de cálcio, composto por pó, um veículo e um radiopacificador, é considerado o melhor medicamento para a deposição de tecido duro e promoção da cicatrização de polpas vitais e tecidos periapicais (Fava, et al., 1999), inclusive para o tratamento endodôntico de dentes que sofreram traumatismo (Astolfi, et al., 2017). Porém, o Calem combinado ao PMCC, inclusive associado a outras substâncias e com concentrações mais diluídas, tem sido proposto por apresentar atividade antimicrobiana mais potente e baixa citotoxidade (Rocha, et al. 2010).

A técnica de instrumentação escolhida para este caso é caracterizada por ser acessível e de baixo custo, podendo ser, inclusive, adotada nos serviços públicos odontológicos e por profissionais não especialistas na área. Assim, foi realizada a técnica híbrida de Tagger que associa a condensação lateral ativa no terço apical com a compactação termomecânica feita com McSpadden (Tagger, et al., 1984), cujo grau de ausência de infiltração apical não difere estatisticamente de outras técnicas de obturação (Carvalho, et al., 2006). Neste caso, o sucesso do tratamento foi constatado após 6 meses e 1 ano de proservação, sem infiltração apical, apresentando como desfecho a regressão total da lesão.

Por fim, a terapia endodôntica conservadora escolhida é considerada a primeira opção para o tratamento deste tipo de lesão periapical (Mitra, et al., 2017; Carneiro, et al., 2020). No entanto, caso haja insucesso no tratamento, a terapia cirúrgica poderá ser necessária como, por exemplo, a marsupialização ou cirurgia paredodôntica. O tratamento cirúrgico necessita ser bem indicado e só será a primeira escolha do profissional caso haja grande risco de fratura dentária durante o acesso ao canal, a estrutura remanescente apresente extenso infiltrado inflamatório agudo e para os casos de lesões de grandes dimensões (Carvalho, et al., 2012; Machionatti, et al., 2017).

Nos casos em que alterações pulpares estão presentes e estas não são tratadas, o que também inclui os casos de traumatismo, não se deve olvidar da possibilidade de necrose pulpar. Dessa forma, o profissional deve ter conhecimento acerca das características histológicas, clínicas e radiográficas dessa patologia para poder reconhecê-las, indicar e/ou conduzir a melhor opção de tratamento (Leonardi, et al., 2011).

\section{Considerações Finais}

Abordagens mais conservadoras devem ser a primeira escolha do profissional mesmo diante de casos complexos, como o apresentado. O correto diagnóstico é imprescindível para a escolha da melhor conduta e o estabelecimento do correto tratamento. 
A escolha de uma técnica mais simples e eficaz mostra que se pode alcançar resultados de excelência tanto quanto técnicas mais complexas e modernas, ou até mesmo mais invasivas.

\section{Referências}

Andreasen, F., \& Kahler, B. (2015). Pulpal response after acute dental injury in the permanent dentition: clinical implications - a review. Journal of endodontics, 41(3), 299-308. 10.1016/j.joen.2014.11.015

Arashiro, F., Ferreira, L., Navarro, \& J.,Tomazinho, L. (2013). Tratamento de canais com instrumentos fraturados: relato de casos. UNINGÁ Review. 14(1), 79-84.

Astolfi, G., Crema, M., Simões, P., \& Ceretta, R. (2017). Tratamento endodôntico em dente desvitalizado por trauma: relato de caso clínico. Rev. Odontol. Univ. Cid. São Paulo, 29(1), 90-9. 10.26843/ro_unicid.v29i1.203

Carneiro, M., Costa, F., Chiora, P., Endo, M., \& Ventrini, V. (2020). Abordagem endodôntica não cirúrgica em extensa lesão periapical: relato de caso. Arch Health Invest, 9(6), 513-516.

Carvalho, E., Júnior, J., Malvar, M., \& Albergaria, S. (2006). Avaliação do selamento apical em dentes obturados pela técnica da condensação lateral híbrida, de Tagger e Thermafil. Rev Cienc méd biol., 5(3), 239-44. 10.9771/cmbio.v5i3.4132

Carvalho, M., Dotto, S., Brondani, G., Filter, V., \& Kist, P. (2012). Reparo de Lesão Periapical: Relato de Caso. Rv de Endodontia Pesquisa e Ensino online, 9,15 .

Fava, L., \& Saunders, W. (1999). Calcium hydroxide pastes: classification and clinical indications. International endodontic jornal, 32(4), 257- 82. 10.1046/j.1365-2591.1999.00232.x

Fouad, A. Microbiological aspects of traumatic injuries. (2019). Dental Traumatology, 35(6), 324-332. doi:10.1111/edt.12494

Leonardi, D., Giovanini, A., Almeida, S., Scramm, C., \& Baratto-Filho, F. (2011). Pulp and periapical pathologies. RSBO, 8(4), e47-61.

Machionatti, A., Wandscher, V., Burmann, P., Tomé, S., \& Wolle, C. (2017). Tratamento cirúrgico de Cisto Periapical de ampla extensão: Relato de Caso. Rv Saúde Integrada, 10, 86-89.

Mitra, A., \& Adhikari, C. (2017). Management of large periapical lesions by non surgical endodontic approach-two case reports. IJIRDS, 2(5), 97-104.

Oliveira E. Irala L., Dos Santos A., \& Melo T. (2010). Avaliação da ação antimicrobiana de quatro formulações à base de hidróxido de cálcio utilizadas como medicação intracanal. $R F O, 15$ (1), 35-39;

Oliveira, L., Salles, L., \& Menezes E. (2019). Tratamento endodôntico conservador de cisto periapical inflamatório. R Odontol Planal Cent., 8 (1), 19-25.

Pereira, A. S., et al. (2018). Metodologia da pesquisa científica. UFSM. https://repositorio.ufsm.br/bitstream/handle/1/15824/Lic_Computacao_MetodologiaPesquisa Cientifica.pdf?sequence $=1$

Petti, S., Andreasen, J., Glendor, U., \& Andersson, L. (2018). The fifth most prevalent disease is being neglected by public health organisations. Lancet Global Health, 6(10), e1070-1. 10.1016/S2214-109X(18)30380-2

Ricucci D., Siqueira J., Loghin S., \& Berman L. (2015). The cracked tooth: histopathologic and histobacteriologic aspects. J Endod, 41 , 343-52.

Rocha, C., Irala, L., Salles, A., \& Soares, R. (2010). Atividade antimicrobiana do PMCC, por contato direto e vapor, frente ao Enterococcus faecalis e ao Staphylococcus aureus. Stomatos, 16(31), 45-54.

Tagger, M., Tamse, A., Katz, A., \& Korzen, B. (1984). Evaluation of the apical seal produced by a hybrid root canal filling method, combining lateral condensation and thermatic compaction. J Endod, 10(7), 299-303. doi:10.1016/S0099-2399(84)80183-1

Vera, J., Siqueira Jr, J., Ricucci, D., Loghin, S., Fernández, N., Flores, B., \& Cruz, A. G. (2012). One-versus two-visit endodontic treatment of teeth with apical periodontitis: a histobacteriologic study. J Endod, 38(8), 1040-1052. doi.org/10.1016/j.joen.2012.04.010

Yamashita, F., Yamashita, I., Pavan, N., \& Endo, M. (2017). Perfil dos pacientes atendidos no Centro de Traumatismo em Odontologia frente às fraturas coronárias e suas sequelas. Archives Of Health Investigation, 6(6). 10.21270/archi.v6i6.2069 